\title{
P-WAVE INTERACTION WITH A PAIR OF RIGID STRIPS EMBEDDED IN AN ORTHOTROPIC STRIP
}

\author{
SANJOY BASU \\ Haldia Institute of Technology, Department of Applied Science, Haldia, India; e-mail: basu1982@gmail.com \\ S.C. MANDAL \\ Jadavpur University, Department of Mathematics, Kolkata, India; e-mail: scmandal@math.jdvu.ac.in
}

\begin{abstract}
The present paper is concerned with the problem of scattering of the $\mathrm{P}$-wave by two co-planer finite rigid strips placed symmetrically in an infinitely long orthotropic strip. Using the Hilbert transform technique, the mixed boundary value problem has been reduced to the solution of dual integral equations which has finally been reduced to the solution of a Fredholm integral equation of the second kind. Solving this integral equation numerically, stress intensity factors have been calculated at the inner and outer edges of the rigid strips, and the vertical displacement outside the strips has been calculated and plotted graphically to show the effect of material orthotropy.
\end{abstract}

Keywords: P-wave, Fourier transform, Hilbert transform, Fredholm integral equation, stress intensity factor

\section{Introduction}

The dynamic interaction of rigid strips with an elastic isotropic or orthotropic medium is a subject of considerable interest in mechanics. Dynamical analysis of this kind is of importance to earth-quake engineering, machine, vibrations and seismology. The performance of engineered systems is affected by inhomogeneities such as cracks and inclusions present in the material. Cracks and rigid inclusions in an elastic material have become the subject of investigations. Presently, the use of anisotropic materials is increasing due to their strength. The increasing use of anisotropic media demands that the study should be extensive. A detailed reference of work done on the determination of the dynamic stress field around a crack or inclusion in an elastic solid was given by Sih (1977), Sih and Chen (1981), Chen (1978), Cinar (1983). However, in the presence of finite boundaries, the problem becomes complicated since they involve additional geometric parameters, describing the dimension of the solids. Forced vertical vibration of a single strip was treated by Wickham (1977). Singh et al. (1983) solved the problem of diffraction of a torsional wave by a circular rigid disc at the interface of two bonded dissimilar elastic solids. In that paper, they discussed an iterative method to solve the Fredholm integral equation of the second kind and described the stress intensity factor with the wave number. Mandal et al. $(1997,1998)$ solved the problem of forced vibration of two and four rigid strips on a semi-infinite elastic medium. Mandal et al. (1998) also treated the diffraction problem by four rigid strips in an orthotropic medium. Interaction of elastic waves with a periodic array of the coplanar Griffith crack in an orthotropic medium was discussed by Mandal et al. (1994). Das et al. (1998) solved the problem of determining the stress intensity factor for an interfacial crack between two orthotropic half planes bonded to a dissimilar orthotropic layer with a punch. They reduced the problem to a system of simultaneous integral equations which were solved by Chebyshev polynomials. The problem of two perfectly bonded dissimilar orthotropic strips with an interfacial crack was studied by Li (2005). He derived the analytical expression for the 
stress intensity factor. Sarkar et al. (1995) solved the problem of diffraction of elastic waves by three coplanar Griffith cracks in an orthotropic medium. Das (2002) solved the problem of interaction between line cracks in an orthotropic layer. An elastostatic problem of an infinite row of parallel cracks in an orthotropic medium was analyzed by Sinharoy (2013). Monfared and Ayatollahi (2013) investigated the problem of determining the dynamic stress intensity factors of multiple cracks in an orthotropic strip with a functionally graded materials coating. They solved the problem by reducing it to a singular integral equation of the Cauchy type. The problem of interaction of three interfacial Griffith cracks between bonded dissimilar orthotropic half planes was studied by Mukherjee and Das (2007). Das et al. (2008) solved the problem of determining the stress intensity factors due to symmetric edge cracks in an orthotropic strip under normal loading. They derived an analytical expression for the stress intensity factor at the crack tip. The problem of finding the stress intensity factors for two parallel interface cracks between a nonhomogeneous bonding layer and two dissimilar orthotropic half-planes under tension was studied by Itou (2012). Shear wave interaction with a pair of rigid strips in elastic strip was analyzed by Pramanick et al. (1999). WU Da-zhi et al. (2006) considered the torsional vibration problem of a rigid circular plate on a transversely isotropic saturated soil. Very recently Morteza et al. $(2010 \mathrm{a}, \mathrm{b})$ considered the vibration problem of a rigid circular disc on transversely isotropic media. Diffraction of elastic waves by two parallel rigid strips in an infinite orthotropic medium was analyzed by Sarkar et al. (1995).

In this paper, the diffraction of the elastic P-wave by two rigid strips embedded in an infinite orthotropic strip is analyzed. Using the Hilbert transform technique, the mixed boundary value problem has been reduced to the Fredholm integral equation of the second kind which has been solved numerically by the Fox and Goodwin method (1953). Stress intensity factors at both the edges of the strips have been calculated and shown graphically for different parameters and materials. Finally, vertical displacement has been calculated outside the strips and shown by 3D-graphs.

\section{Formulation of the problem}

Let us consider an infinitely long orthotropic elastic strip of width $2 h$ containing two coplanar rigid strips embedded in it. The location of the strips are $b \leqslant|X| \leqslant a, Y=0,|Z|<\infty$, with reference to the cartesian co-ordinate axes $(X, Y, Z)$. Normalizing all lengths with respect to $a$ and putting $X / a=x, Y / a=y, Z / a=z, b / a=c$, the locations of the rigid strips are defined by $c \leqslant|x| \leqslant 1, y=0,|z|<\infty$ (Fig. 1 ).

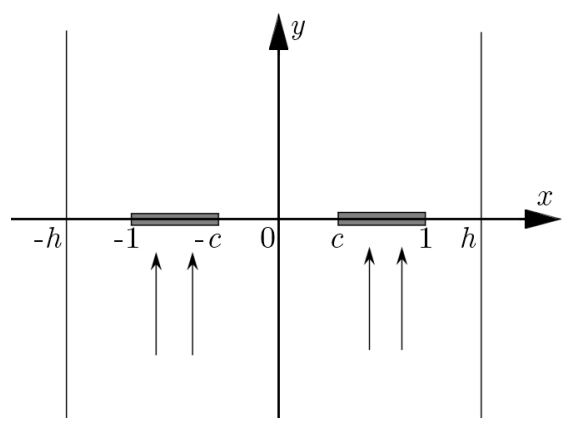

Fig. 1. Geometry of the strips

Let a time harmonic wave given by $u=0$ and $v=v_{0} \mathrm{e}^{\mathrm{i}(k y-\omega t)}$, where $k=a \omega /\left(c_{s} \sqrt{c_{22}}\right)$, $c_{s}=\sqrt{\mu_{12} / \rho}$ with $\rho$ being the density of the material, $\omega$ the circular frequency and $v_{0}$ a constant, travelling in the direction of the positive $y$-axis and be incident normally on the strips. 
The non-zero stress components $\tau_{y y}, \tau_{x y}$ and $\tau_{x x}$ are given by

$$
\frac{\tau_{y y}}{\mu_{12}}=c_{12} \frac{\partial u}{\partial x}+c_{22} \frac{\partial v}{\partial y} \quad \frac{\tau_{x y}}{\mu_{12}}=\frac{\partial u}{\partial y}+\frac{\partial v}{\partial x} \quad \frac{\tau_{x x}}{\mu_{12}}=c_{11} \frac{\partial u}{\partial x}+c_{12} \frac{\partial v}{\partial y}
$$

where $u$ and $v$ are displacement components and $c_{i j}(i, j=1,2)$ are non-dimensional parameters related to the engineering elastic constants $E_{i}, \mu_{i j}$ and $\nu_{i j}(i, j=1,2,3)$ by the relations

$$
\begin{aligned}
& c_{11}=\frac{E_{1}}{\mu_{12}}\left(1-\frac{\nu_{12}^{2} E_{2}}{E_{1}}\right) \quad c_{22}=\frac{E_{2}}{\mu_{12}}\left(1-\frac{\nu_{12}^{2} E_{2}}{E_{1}}\right)=c_{11} \frac{E_{2}}{E_{1}} \\
& c_{12}=\frac{\nu_{12} E_{2}}{\mu_{12}}\left(1-\frac{\nu_{12}^{2} E_{2}}{E_{1}}\right)=\nu_{12} c_{22}=\nu_{21} c_{11}
\end{aligned}
$$

for the generalized plane stress and

$$
\begin{aligned}
& c_{11}=\frac{E_{1}}{\Delta \mu_{12}}\left(1-\nu_{23} \nu_{32}\right) \quad c_{22}=\frac{E_{2}}{\Delta \mu_{12}}\left(1-\nu_{13} \nu_{31}\right) \\
& c_{12}=\frac{E_{1}}{\Delta \mu_{12}}\left(\nu_{21}+\frac{\nu_{13} \nu_{32} E_{2}}{E_{1}}\right)=\frac{E_{2}}{\Delta \mu_{12}}\left(\nu_{12}+\frac{\nu_{23} \nu_{31} E_{1}}{E_{2}}\right)
\end{aligned}
$$

where

$$
\Delta=1-\nu_{12} \nu_{21}-\nu_{23} \nu_{32}-\nu_{31} \nu_{13}-\nu_{12} \nu_{23} \nu_{31}-\nu_{13} \nu_{21} \nu_{32}
$$

for the plane strain. The constants $E_{i}$ and $\nu_{i j}$ satisfy Maxwell's relation

$$
\frac{\nu_{i j}}{E_{i}}=\frac{\nu_{j i}}{E_{j}}
$$

Therefore, substituting $u(x, y, t)=u(x, y) \mathrm{e}^{-\mathrm{i} \omega t}$ and $v(x, y, t)=v(x, y) \mathrm{e}^{-\mathrm{i} \omega t}$, our problem reduces to the solution of the equations

$$
\begin{aligned}
& c_{11} \frac{\partial^{2} u}{\partial x^{2}}+\frac{\partial^{2} u}{\partial y^{2}}+\left(1+c_{12}\right) \frac{\partial^{2} v}{\partial x \partial y}+k_{s}^{2} u=0 \\
& c_{22} \frac{\partial^{2} v}{\partial y^{2}}+\frac{\partial^{2} v}{\partial x^{2}}+\left(1+c_{12}\right) \frac{\partial^{2} u}{\partial x \partial y}+k_{s}^{2} v=0
\end{aligned}
$$

where $k_{s}^{2}=a^{2} \omega^{2} / c_{s}^{2}$.

Thus the problem is to find the stress distribution near the edges of the strips subject to the following boundary conditions

$$
\begin{array}{lll}
v(x, 0+)=v(x, 0-)=-v_{0} & c \leqslant|x| \leqslant 1 \\
\tau_{y y}(x, 0)=0 & |x|<c & 1<|x|<h \\
u(x, 0)=0 & |x|<h & \\
\tau_{x x}( \pm h, y)=0 & \tau_{x y}( \pm h, y)=0
\end{array}
$$

Henceforth, the time factor $\mathrm{e}^{-\mathrm{i} \omega t}$ which is common to all field variables will be omitted in the sequel. 
The solution to equations (2.5) can be taken as

$$
\begin{aligned}
& u(x, y)=\frac{2}{\pi} \int_{0}^{\infty}\left[A_{1}(\xi) \mathrm{e}^{-\nu_{1}|y|}+A_{2}(\xi) \mathrm{e}^{-\nu_{2}|y|}\right] \sin (\xi x) d \xi \\
&+\frac{2}{\pi} \int_{0}^{\infty}\left[A_{3}(\zeta) \sinh \left(\nu_{3} x\right)+A_{4}(\zeta) \sinh \left(\nu_{4} x\right)\right] \sin (\zeta y) d \zeta \\
& v(x, y)=\frac{2}{\pi} \int_{0}^{\infty} \frac{1}{\xi}\left[\alpha_{1} A_{1}(\xi) \mathrm{e}^{-\nu_{1}|y|}+\alpha_{2} A_{2}(\xi) \mathrm{e}^{-\nu_{2}|y|}\right] \cos (\xi x) d \xi \\
&+\frac{2}{\pi} \int_{0}^{\infty} \frac{1}{\zeta}\left[\alpha_{3} A_{3}(\zeta) \cosh \left(\nu_{3} x\right)+\alpha_{4} A_{4}(\zeta) \cosh \left(\nu_{4} x\right)\right] \cos (\zeta y) d \zeta
\end{aligned}
$$

where $A_{i}(\xi)(i=1,2,3,4)$ are the unknown functions to be determined, $\nu_{1}^{2}$ and $\nu_{2}^{2}$ are the roots of the equation

$$
c_{22} \nu^{4}+\left\{\left(c_{12}^{2}+2 c_{12}-c_{11} c_{22}\right) \xi^{2}+\left(1+c_{22}\right) k_{s}^{2}\right\} \nu^{2}+\left(c_{11} \xi^{2}-k_{s}^{2}\right)\left(\xi^{2}-k_{s}^{2}\right)=0
$$

and $\nu_{3}^{2}, \nu_{4}^{2}$ are the roots of the equation

$$
c_{11} \nu^{4}+\left\{\left(c_{12}^{2}+2 c_{12}-c_{11} c_{22}\right) \zeta^{2}+\left(1+c_{11}\right) k_{s}^{2}\right\} \nu^{2}+\left(c_{22} \zeta^{2}-k_{s}^{2}\right)\left(\zeta^{2}-k_{s}^{2}\right)=0
$$

where

$$
\alpha_{i}= \begin{cases}\frac{c_{11} \xi^{2}-k_{s}^{2}-\nu_{i}^{2}}{\left(1+c_{12}\right) \nu_{i}} & i=1,2 \\ \frac{\zeta^{2}-k_{s}^{2}-c_{11} \nu_{i}^{2}}{\left(1+c_{12}\right) \nu_{i}} & i=3,4\end{cases}
$$

From boundary condition (2.11), it is found that

$$
A_{2}(\xi)=-A_{1}(\xi)
$$

Therefore, the displacements $u, v$ and stresses $\tau_{y y}, \tau_{x y}, \tau_{x x}$ can be finally written as

$$
\begin{aligned}
& u(x, y)=\frac{2}{\pi} \int_{0}^{\infty}\left[\mathrm{e}^{-\nu_{1}|y|}-\mathrm{e}^{-\nu_{2}|y|}\right] A_{1}(\xi) \sin (\xi x) d \xi \\
&+\frac{2}{\pi} \int_{0}^{\infty}\left[A_{3}(\zeta) \sinh \left(\nu_{3} x\right)+A_{4}(\zeta) \sinh \left(\nu_{4} x\right)\right] \sin (\zeta y) d \zeta \\
& v(x, y)=\frac{2}{\pi} \int_{0}^{\infty} \frac{1}{\xi}\left[\alpha_{1} \mathrm{e}^{-\nu_{1}|y|}-\alpha_{2} \mathrm{e}^{-\nu_{2}|y|}\right] A_{1}(\xi) \cos (\xi x) d \xi \\
&+\frac{2}{\pi} \int_{0}^{\infty} \frac{1}{\zeta}\left[\alpha_{3} A_{3}(\zeta) \cosh \left(\nu_{3} x\right)+\alpha_{4} A_{4}(\zeta) \cosh \left(\nu_{4} x\right)\right] \cos (\zeta y) d \zeta
\end{aligned}
$$


and

$$
\begin{aligned}
\frac{\tau_{y y}}{\mu_{12}} & =\frac{2}{\pi}\left\{\int_{0}^{\infty}\left[\left(c_{12} \xi-\operatorname{sgn}(y) \frac{c_{22} \alpha_{1} \nu_{1}}{\xi}\right) \mathrm{e}^{-\nu_{1}|y|}-\left(c_{12} \xi-\operatorname{sgn}(y) \frac{c_{22} \alpha_{2} \nu_{2}}{\xi}\right) \mathrm{e}^{-\nu_{2}|y|}\right]\right. \\
& \cdot A_{1}(\xi) \cos (\xi x) d \xi+\int_{0}^{\infty}\left[\left(c_{12} \nu_{3}-c_{22} \alpha_{3}\right) A_{3}(\zeta) \cosh \left(\nu_{3} x\right)\right. \\
& \left.\left.+\left(c_{12} \nu_{4}-c_{22} \alpha_{4}\right) A_{4}(\zeta) \cosh \left(\nu_{4} x\right)\right] \sin (\zeta y) d \zeta\right\} \\
\frac{\tau_{x y}}{\mu_{12}} & =-\frac{2}{\pi}\left\{\int_{0}^{\infty}\left[\left(\nu_{1}+\alpha_{1}\right) \mathrm{e}^{-\nu_{1} y}-\left(\nu_{2}+\alpha_{2}\right) \mathrm{e}^{-\nu_{2} y}\right] A_{1}(\xi) \sin (\xi x) d \xi\right. \\
& +\int_{0}^{\infty}\left[\left(\zeta+\frac{\nu_{3} \alpha_{3}}{\zeta}\right) A_{3}(\zeta) \sinh \left(\nu_{3} x\right)\right. \\
& \left.\left.+\left(\zeta+\frac{\nu_{4} \alpha_{4}}{\zeta}\right) A_{4}(\zeta) \sinh \left(\nu_{4} x\right)\right] \cos (\zeta y) d \zeta\right\} \\
\frac{\tau_{x x}}{\mu_{12}} & =\frac{2}{\pi}\left\{\int_{0}^{\infty}\left[\left(c_{11} \xi-\frac{c_{12} \alpha_{1} \nu_{1}}{\xi}\right) \mathrm{e}^{-\nu_{1}|y|}-\left(c_{11} \xi-\frac{c_{12} \alpha_{2} \nu_{2}}{\xi}\right) \mathrm{e}^{-\nu_{2}|y|}\right] A_{1}(\xi) \cos (\xi x) d \xi\right. \\
& +\int_{0}^{\infty}\left[\left(c_{11} \nu_{3}-c_{12} \alpha_{3}\right) A_{3}(\zeta) \cosh \left(\nu_{3} x\right)\right. \\
& \left.\left.+\left(c_{11} \nu_{4}-c_{12} \alpha_{4}\right) A_{4}(\zeta) \cosh \left(\nu_{4} x\right)\right] \sin (\zeta y) d \zeta\right\} \quad y>0
\end{aligned}
$$

Boundary conditions (2.6) and (2.7) yield the following pair of dual integral equations

$$
\begin{aligned}
& \int_{0}^{\infty} \frac{1}{\xi}[1+H(\xi)] A(\xi) \cos (\xi x) d \xi=p(x) \quad c \leqslant|x| \leqslant 1 \\
& \int_{0}^{\infty} A(\xi) \cos (\xi x) d \xi=0 \quad|x|<c \quad 1<|x|<h
\end{aligned}
$$

where

$$
\begin{aligned}
& A(\xi)=\frac{\alpha_{1} \nu_{1}-\alpha_{2} \nu_{2}}{\xi} A_{1}(\xi) \\
& H(\xi)=\left(\frac{\alpha_{1}-\alpha_{2}}{\alpha_{1} \nu_{1}-\alpha_{2} \nu_{2}}\right) \frac{\xi}{d}-1 \rightarrow 0 \quad \text { as } \xi \rightarrow \infty \\
& p(x)=-\frac{\pi}{2 c} v_{0}-\frac{1}{c} \int_{0}^{\infty} \frac{1}{\zeta}\left[\alpha_{3} A_{3}(\zeta) \cosh \left(\nu_{3} x\right)+\alpha_{4} A_{4}(\zeta) \cosh \left(\nu_{4} x\right)\right] d \zeta \\
& d=\frac{c_{11}+N_{1} N_{2}}{N_{1} N_{2}\left(N_{1}+N_{2}\right)}
\end{aligned}
$$

and

$$
\begin{aligned}
& N_{1}^{2}=\frac{1}{2 c_{22}}\left[-\left(c_{12}^{2}+2 c_{12}-c_{11} c_{22}\right)+\sqrt{\left(c_{12}^{2}+2 c_{12}-c_{11} c_{22}\right)^{2}-4 c_{11} c_{22}}\right] \\
& N_{2}^{2}=\frac{1}{2 c_{22}}\left[-\left(c_{12}^{2}+2 c_{12}-c_{11} c_{22}\right)-\sqrt{\left(c_{12}^{2}+2 c_{12}-c_{11} c_{22}\right)^{2}-4 c_{11} c_{22}}\right]
\end{aligned}
$$


Using boundary conditions $(2.9), A_{3}(\zeta)$ and $A_{4}(\zeta)$ are expressed in terms of the function $A(\xi)$ as

$$
\begin{aligned}
& M(\zeta) A_{3}(\zeta)=\left(\zeta+\frac{\alpha_{4} \nu_{4}}{\zeta}\right) i_{1}(\zeta) \sinh \left(\nu_{4} h\right)-\left(c_{11} \nu_{4}-c_{12} \alpha_{4}\right) i_{2}(\zeta) \cosh \left(\nu_{4} h\right) \\
& M(\zeta) A_{4}(\zeta)=-\left(\zeta+\frac{\alpha_{3} \nu_{3}}{\zeta}\right) i_{1}(\zeta) \sinh \left(\nu_{3} h\right)+\left(c_{11} \nu_{3}-c_{12} \alpha_{3}\right) i_{2}(\zeta) \cosh \left(\nu_{3} h\right)
\end{aligned}
$$

where

$$
\begin{gathered}
M(\zeta)=\left(\zeta+\frac{\alpha_{4} \nu_{4}}{\zeta}\right)\left(c_{11} \nu_{3}-c_{12} \alpha_{3}\right) \cosh \left(\nu_{3} h\right) \sinh \left(\nu_{4} h\right) \\
-\left(\zeta+\frac{\alpha_{3} \nu_{3}}{\zeta}\right)\left(c_{11} \nu_{4}-c_{12} \alpha_{4}\right) \sinh \left(\nu_{3} h\right) \cosh \left(\nu_{4} h\right)
\end{gathered}
$$

and

$$
\begin{aligned}
& i_{1}(\zeta)=\frac{2}{\pi} \int_{0}^{\infty}\left\{\frac{\zeta\left[c_{11} \xi^{2}+c_{12}\left(k_{s}^{2}+\nu_{1}^{2}\right)\right]}{\nu_{1}^{2}+\zeta^{2}}-\frac{\zeta\left[c_{11} \xi^{2}+c_{12}\left(k_{s}^{2}+\nu_{2}^{2}\right)\right]}{\nu_{2}^{2}+\zeta^{2}}\right\} \frac{A(\xi) \cos (\xi h)}{\nu_{1}^{2}-\nu_{2}^{2}} d \xi \\
& i_{2}(\zeta)=-\frac{2}{\pi} \int_{0}^{\infty}\left(\frac{c_{12} \nu_{1}^{2}+c_{11} \xi^{2}-k_{s}^{2}}{\nu_{1}^{2}+\zeta^{2}}-\frac{c_{12} \nu_{2}^{2}+c_{11} \xi^{2}-k_{s}^{2}}{\nu_{2}^{2}+\zeta^{2}}\right) \frac{\xi A(\xi) \sin (\xi h)}{\nu_{1}^{2}-\nu_{2}^{2}} d \xi
\end{aligned}
$$

\section{Method of solution}

In order to reduce dual integral equations (2.17) to a single Fredholm integral equation, let us assume that

$$
A(\xi)=\int_{c}^{1} \frac{h\left(t^{2}\right)}{t}[1-\cos (\xi t)] d t
$$

where the unknown function $h\left(t^{2}\right)$ is to be determined.

Substituting $A(\xi)$ from (3.1) into equations $(2.17)_{2}$, we note that

$$
\int_{0}^{\infty} A(\xi) \cos (\xi x) d \xi=\pi \int_{c}^{1} \frac{h\left(t^{2}\right)}{t}\left[\delta(x)-\frac{1}{2} \delta(x+t)-\frac{1}{2} \delta(|x-t|)\right] d t
$$

so that equation $(2.17)_{2}$ is automatically satisfied.

Again, the substitution of the value of $A(\xi)$ from (3.1) into equation (2.17) 1 yields

$$
\frac{1}{2} \int_{c}^{1} \frac{h\left(t^{2}\right)}{t} \log \left|\frac{x^{2}-t^{2}}{x^{2}}\right| d t=p(x)-\int_{c}^{1} \frac{h\left(t^{2}\right)}{t} d t \int_{0}^{\infty} \xi^{-1} H(\xi) \cos (\xi x)[1-\cos (\xi t)] d \xi
$$

Differentiating both sides of equation (3.2) with respect to $x$ and subsequently multiplying by $(-2 x / \pi)$, we obtain

$$
\begin{aligned}
& \frac{2}{\pi} \int_{c}^{1} \frac{t h\left(t^{2}\right)}{t^{2}-x^{2}} d t \\
& \quad=\frac{2 x}{\pi} \int_{c}^{1} \frac{h\left(t^{2}\right)}{t} d t\left\{\frac{1}{d} \int_{0}^{\infty} \frac{1}{\zeta}\left[\alpha_{3} \nu_{3} A_{5}(\zeta) \sinh \left(\nu_{3} x\right)+\alpha_{4} \nu_{4} A_{6}(\zeta) \sinh \left(\nu_{4} x\right)\right] d \zeta\right. \\
& \left.\quad-\int_{0}^{\infty} H(\xi) \sin (\xi x)[1-\cos (\xi t)] d \xi\right\} \quad c \leqslant|x| \leqslant 1
\end{aligned}
$$


Using the Hilbert transform technique, the solution to integral equation (3.3) is given by

$$
h\left(u^{2}\right)+\int_{c}^{1} \frac{h\left(t^{2}\right)}{t}\left[k_{1}\left(u^{2}, t^{2}\right)+k_{2}\left(u^{2}, t^{2}\right)\right] d t=\frac{D}{\sqrt{\left(u^{2}-c^{2}\right)\left(1-u^{2}\right)}}
$$

where

$$
\begin{aligned}
& k_{1}\left(u^{2}, t^{2}\right)=\frac{4}{\pi^{2} d} \sqrt{\frac{u^{2}-c^{2}}{1-u^{2}}} \int_{c}^{1} \sqrt{\frac{1-x^{2}}{x^{2}-c^{2}}} \frac{x^{2}}{x^{2}-u^{2}} d x \\
& \cdot\left\{\int_{0}^{\infty} \frac{1}{\zeta}\left[\alpha_{3} \nu_{3} A_{5}(\zeta) \sinh \left(\nu_{3} x\right)+\alpha_{4} \nu_{4} A_{6}(\zeta) \sinh \left(\nu_{4} x\right)\right] d \zeta\right\} \\
& k_{2}\left(u^{2}, t^{2}\right)=-\frac{4}{\pi^{2}} \sqrt{\frac{u^{2}-c^{2}}{1-u^{2}}} \int_{c}^{1} \sqrt{\frac{1-x^{2}}{x^{2}-c^{2}}} \frac{x^{2} d x}{x^{2}-u^{2}} \int_{0}^{\infty} H(\xi) \sin (\xi x)[1-\cos (\xi t)] d \xi \\
& A_{5}(\zeta)=\frac{1}{M(\zeta)}\left[\left(\zeta+\frac{\alpha_{4} \nu_{4}}{\zeta}\right) i_{3}(\zeta) \sinh \left(\nu_{4} h\right)-\left(c_{11} \nu_{4}-c_{12} \alpha_{4}\right) i_{4}(\zeta) \cosh \left(\nu_{4} h\right)\right] \\
& A_{6}(\zeta)=-\frac{1}{M(\zeta)}\left[\left(\zeta+\frac{\alpha_{3} \nu_{3}}{\zeta}\right) i_{3}(\zeta) \sinh \left(\nu_{3} h\right)+\left(c_{11} \nu_{3}-c_{12} \alpha_{3}\right) i_{4}(\zeta) \cosh \left(\nu_{3} h\right)\right]
\end{aligned}
$$

and

$$
\begin{aligned}
& i_{3}(\zeta)=\frac{2}{\pi} \int_{0}^{\infty}\left\{\frac{\zeta\left[c_{11} \xi^{2}+c_{12}\left(k_{s}^{2}+\nu_{1}^{2}\right)\right]}{\nu_{1}^{2}+\zeta^{2}}-\frac{\zeta\left[c_{11} \xi^{2}+c_{12}\left(k_{s}^{2}+\nu_{2}^{2}\right)\right]}{\nu_{2}^{2}+\zeta^{2}}\right\} \frac{[1-\cos (\xi t)] \cos (\xi h)}{\nu_{1}^{2}-\nu_{2}^{2}} d \xi \\
& i_{4}(\zeta)=-\frac{2}{\pi} \int_{0}^{\infty}\left(\frac{c_{12} \nu_{1}^{2}+c_{11} \xi^{2}-k_{s}^{2}}{\nu_{1}^{2}+\zeta^{2}}-\frac{c_{12} \nu_{2}^{2}+c_{11} \xi^{2}-k_{s}^{2}}{\nu_{2}^{2}+\zeta^{2}}\right) \frac{\xi[1-\cos (\xi t)]}{\nu_{1}^{2}-\nu_{2}^{2}} \sin (\xi h) d \xi
\end{aligned}
$$

In order to determine the arbitrary constant $D$, multiplying equation (3.2) by $x / \sqrt{\left(x^{2}-c^{2}\right)\left(1-x^{2}\right)}$ and integrating from $c$ to 1 with respect to $x$, we obtain

$$
\begin{aligned}
& \int_{c}^{1} \frac{h\left(u^{2}\right)}{u} d u=-\frac{\pi v_{0}}{c \log \left|\frac{1-c}{1+c}\right|}-\frac{4}{\pi \log \left|\frac{1-c}{1+c}\right|}\left[\int_{c}^{1} \frac{x B_{1}\left(x, t^{2}\right)}{\sqrt{\left(x^{2}-c^{2}\right)\left(1-x^{2}\right)}} d x\right. \\
& \left.\quad+\int_{c}^{1} \frac{h\left(t^{2}\right)}{t} d t \int_{c}^{1} \frac{x B_{2}\left(x, t^{2}\right)}{\sqrt{\left(x^{2}-c^{2}\right)\left(1-x^{2}\right)}} d x\right]
\end{aligned}
$$

where

$$
\begin{aligned}
& B_{1}\left(x, t^{2}\right)=\frac{1}{d} \int_{0}^{\infty} \frac{1}{\zeta}\left[\alpha_{3} A_{5}(\zeta) \cosh \left(\nu_{3} x\right)+\alpha_{4} A_{6}(\zeta) \cosh \left(\nu_{4} x\right)\right] d \zeta \\
& B_{2}\left(x, t^{2}\right)=\int_{0}^{\infty} \frac{1}{\xi} H(\xi) \cos (\xi x)[1-\cos (\xi t)] d \xi
\end{aligned}
$$

Again, substituting $h\left(u^{2}\right)$ from equation (3.4) into equation (3.7) and simplifying, we obtain

$$
\begin{aligned}
D= & -\frac{2 v_{0} c}{d \log \left|\frac{1-c}{1+c}\right|}-\frac{8 c}{\pi^{2} \log \left|\frac{1-c}{1+c}\right|} \int_{c}^{1} \frac{h\left(t^{2}\right)}{t} d t \int_{c}^{1} \frac{x\left[B_{1}\left(x, t^{2}\right)+B_{2}\left(x, t^{2}\right)\right]}{\sqrt{\left(x^{2}-c^{2}\right)\left(1-x^{2}\right)}} d x \\
& +\frac{2 c}{\pi} \int_{c}^{1} \frac{h\left(t^{2}\right)}{t} d t \int_{c}^{1} \frac{1}{u}\left[k_{1}\left(u^{2}, t^{2}\right)+k_{2}\left(u^{2}, t^{2}\right)\right] d u
\end{aligned}
$$


Eliminating $D$ from equations (3.4) and (3.9) and simplifying, we obtain

$$
\begin{aligned}
& \sqrt{\left(u^{2}-c^{2}\right)\left(1-u^{2}\right)} h\left(u^{2}\right)+\int_{c}^{1} \frac{h\left(t^{2}\right)}{t}\left[k_{a}\left(u^{2}, t^{2}\right)+k_{b}\left(u^{2}, t^{2}\right)+k_{c}\left(u^{2}, t^{2}\right)\right] d t \\
& =-\frac{2 v_{0} c}{d \log \left|\frac{1-c}{1+c}\right|}
\end{aligned}
$$

where

$$
\begin{aligned}
& k_{a}\left(u^{2}, t^{2}\right)=\frac{4}{\pi^{2}}\left(u^{2}-c^{2}\right) \int_{c}^{1} \sqrt{\frac{1-x^{2}}{x^{2}-c^{2}}} \frac{x^{2}}{x^{2}-u^{2}}\left\{\frac{\partial}{\partial x}\left[B_{1}\left(x, t^{2}\right)+B_{2}\left(x, t^{2}\right)\right]\right\} d x \\
& k_{b}\left(u^{2}, t^{2}\right)=\frac{8 c}{\pi^{2} \log \left|\frac{1-c}{1+c}\right|} \int_{c}^{1} \frac{x\left[B_{1}\left(x, t^{2}\right)+B_{2}\left(x, t^{2}\right)\right]}{\sqrt{\left(x^{2}-c^{2}\right)\left(1-x^{2}\right)}} d x \\
& k_{c}\left(u^{2}, t^{2}\right)=-\frac{8 c}{\pi^{3} u} \sqrt{\frac{u^{2}-c^{2}}{1-u^{2}}} \int_{c}^{1} \sqrt{\frac{1-x^{2}}{x^{2}-c^{2}}} \frac{x^{2}}{x^{2}-u^{2}}\left\{\frac{\partial}{\partial x}\left[B_{1}\left(x, t^{2}\right)+B_{2}\left(x, t^{2}\right)\right]\right\} d x
\end{aligned}
$$

Next, for further simplification, we put

$$
\sqrt{\left(u^{2}-c^{2}\right)\left(1-u^{2}\right)} h\left(u^{2}\right)=H\left(u^{2}\right)
$$

and make the substitution

$$
u^{2}=c^{2} \cos ^{2} \phi+\sin ^{2} \phi \quad t^{2}=c^{2} \cos ^{2} \theta+\sin ^{2} \theta
$$

into equation (3.10) which then reduces to the form

$$
G(\phi)+\int_{0}^{\frac{\pi}{2}} \frac{G(\theta)}{c^{2} \cos ^{2} \theta+\sin ^{2} \theta}\left[k_{a}^{\prime}(\phi, \theta)+k_{b}^{\prime}(\phi, \theta)+k_{c}^{\prime}(\phi, \theta)\right] d \theta=-\frac{2 v_{0} c}{d \log \left|\frac{1-c}{1+c}\right|}
$$

where

$$
\begin{aligned}
& G(\phi)=H\left(c^{2} \cos ^{2} \phi+\sin ^{2} \phi\right) \\
& G(\theta)=H\left(c^{2} \cos ^{2} \theta+\sin ^{2} \theta\right) \\
& k_{a}^{\prime}(\phi, \theta)=k_{a}\left(c^{2} \cos ^{2} \phi+\sin ^{2} \phi, c^{2} \cos ^{2} \theta+\sin ^{2} \theta\right) \\
& k_{b}^{\prime}(\phi, \theta)=k_{b}\left(c^{2} \cos ^{2} \phi+\sin ^{2} \phi, c^{2} \cos ^{2} \theta+\sin ^{2} \theta\right) \\
& k_{c}^{\prime}(\phi, \theta)=k_{c}\left(c^{2} \cos ^{2} \phi+\sin ^{2} \phi, c^{2} \cos ^{2} \theta+\sin ^{2} \theta\right)
\end{aligned}
$$

When $h$ tends to infinity $(h \rightarrow \infty)$, the medium becomes infinite. In this case, the expression for $p(x)$ given by equation $(2.18)_{3}$ becomes $p(x)=-(\pi / 2 c) v_{0}$, since $A_{3}(\zeta)$ and $A_{4}(\zeta)$ given by equations (2.20)-(2.22) become zero.

$A_{3}(\zeta)$ can be written as

$$
A_{3}(\zeta)=\frac{1}{2 M(\zeta)}\left[\left(\zeta+\frac{\alpha_{4} \nu_{4}}{\zeta}\right) i_{1}(\zeta)\left(\mathrm{e}^{\nu_{4} h}-\mathrm{e}^{-\nu_{4} h}\right)-\left(c_{11} \nu_{4}-c_{12} \alpha_{4}\right) i_{2}(\zeta)\left(\mathrm{e}^{\nu_{4} h}+\mathrm{e}^{-\nu_{4} h}\right)\right]
$$

where

$$
\begin{gathered}
M(\zeta)=\frac{1}{4}\left[\left(\zeta+\frac{\alpha_{4} \nu_{4}}{\zeta}\right)\left(c_{11} \nu_{3}-c_{12} \alpha_{3}\right)\left(\mathrm{e}^{\nu_{3} h}+\mathrm{e}^{-\nu_{3} h}\right)\left(\mathrm{e}^{\nu_{4} h}-\mathrm{e}^{-\nu_{4} h}\right)\right. \\
\left.-\left(\zeta+\frac{\alpha_{3} \nu_{3}}{\zeta}\right)\left(c_{11} \nu_{4}-c_{12} \alpha_{4}\right)\left(\mathrm{e}^{\nu_{3} h}-\mathrm{e}^{-\nu_{3} h}\right)\left(\mathrm{e}^{\nu_{4} h}+\mathrm{e}^{-\nu_{4} h}\right)\right]
\end{gathered}
$$


Therefore,

$$
A_{3}(\zeta)=\frac{1}{M_{1}(\zeta)}\left[\left(\zeta+\frac{\alpha_{4} \nu_{4}}{\zeta}\right) i_{1}(\zeta)\left(1-\mathrm{e}^{-2 \nu_{4} h}\right)-\left(c_{11} \nu_{4}-c_{12} \alpha_{4}\right) i_{2}(\zeta)\left(1+\mathrm{e}^{-2 \nu_{4} h}\right)\right]
$$

and

$$
\begin{gathered}
M_{1}(\zeta)=\frac{\mathrm{e}^{\nu_{3}} h}{2}\left[\left(\zeta+\frac{\alpha_{4} \nu_{4}}{\zeta}\right)\left(c_{11} \nu_{3}-c_{12} \alpha_{3}\right)\left(1+\mathrm{e}^{-2 \nu_{3} h}\right)\left(1-\mathrm{e}^{-2 \nu_{4} h}\right)\right. \\
\left.-\left(\zeta+\frac{\alpha_{3} \nu_{3}}{\zeta}\right)\left(c_{11} \nu_{4}-c_{12} \alpha_{4}\right)\left(1-\mathrm{e}^{-2 \nu_{3} h}\right)\left(1+\mathrm{e}^{-2 \nu_{4} h}\right)\right]
\end{gathered}
$$

As $h \rightarrow \infty, M_{1}(\zeta) \rightarrow \infty$ and therefore $A_{3}(\zeta) \rightarrow 0$. Similarly, $A_{4}(\zeta) \rightarrow 0$.

So in this case, dual integral equations $(2.17)_{1}$ and $(2.17)_{2}$ become

$$
\begin{array}{lll}
\int_{0}^{\infty} \frac{1}{\xi}[1+H(\xi)] A(\xi) \cos (\xi x) d \xi=-\frac{\pi}{2 c} v_{0} & & c \leqslant|x| \leqslant 1 \\
\int_{0}^{\infty} A(\xi) \cos (\xi x) d \xi=0 & |x|<c & |x|>1
\end{array}
$$

This problem has been analyzed in detail by Sarkar et al. (1995).

\section{Quantities of physical interest}

The stress $\tau_{y y}(x, y)$ for $y \rightarrow 0$ in the neighbourhood of the strip can be found from equation $(2.16)_{1}$, and is given by

$$
\tau_{y y}(x, 0 \pm)=\mp \frac{2 \mu_{12} c_{22}}{\pi} \int_{0}^{\infty} A(\xi) \cos (\xi x) d \xi \quad c \leqslant|x| \leqslant 1
$$

Now

$$
\Delta \tau_{y y}(x, 0)=\tau_{y y}(x, 0+)-\tau_{y y}(x, 0-)
$$

then

$$
\Delta \tau_{y y}(x, 0)=-\frac{4}{\pi} \mu_{12} c_{22} \int_{0}^{\infty} A(\xi) \cos (\xi x) d \xi
$$

Substituting the value of $A(\xi)$ from equation (3.1) into equation (4.3), we get

$$
\Delta \tau_{y y}(x, 0)=2 \mu_{12} c_{22} \frac{h\left(x^{2}\right)}{x}
$$

Since

$$
h\left(x^{2}\right)=\frac{1}{\sqrt{\left(x^{2}-c^{2}\right)\left(1-x^{2}\right)}} H\left(x^{2}\right) \quad x^{2}=c^{2} \cos ^{2} \phi+\sin ^{2} \phi
$$

equation (4.4) becomes

$$
\Delta \tau_{y y}(x, 0)=\frac{2 \mu_{12} c_{22} G(\phi)}{x \sqrt{\left(x^{2}-c^{2}\right)\left(1-x^{2}\right)}}
$$


So the stress intensity factors $N_{c}$ and $N_{1}$ at the two tips of the strip can be expressed as

$$
N_{c}=\lim _{x \rightarrow c+}\left[\frac{\Delta \tau_{y y}(x, 0)}{\pi c_{22} \mu_{12}} \sqrt{x-c}\right]=\frac{2}{\pi} \frac{G(0)}{c \sqrt{2 c\left(1-c^{2}\right)}}
$$

and

$$
N_{1}=\lim _{x \rightarrow 1-}\left[\frac{\Delta \tau_{y y}(x, 0)}{\pi c_{22} \mu_{12}} \sqrt{1-x}\right]=\frac{2}{\pi} \frac{G\left(\frac{\pi}{2}\right)}{\sqrt{2\left(1-c^{2}\right)}}
$$

Making $c$ tend to zero, the two strips merge into one, and in that case

$$
N_{1}=\frac{\sqrt{2}}{\pi} G\left(\frac{\pi}{2}\right)
$$

Now from equation $(2.15)_{2}$ after substituting the value of $A_{1}(\xi)$ and using equation (3.1), we get the vertical displacement outside the strip as

$$
\begin{aligned}
& v(x, y)=\frac{2}{\pi} \int_{c}^{1} \frac{h\left(t^{2}\right)}{t} d t\left\{\int_{0}^{\infty}\left(\alpha_{1} \mathrm{e}^{-\nu_{1} y}-\alpha_{2} \mathrm{e}^{-\nu_{2} y}\right) \frac{[1-\cos (\xi t)] \cos (\xi x)}{\alpha_{1} \nu_{1}-\alpha_{2} \nu_{2}} d \xi\right. \\
& \left.+\int_{0}^{\infty} \frac{1}{\zeta}\left[\alpha_{3} A_{5}(\zeta) \cosh \left(\nu_{3} x\right)+\alpha_{4} A_{6}(\zeta) \cosh \left(\nu_{4} x\right)\right] \cos (\zeta y) d \zeta\right\}
\end{aligned}
$$

\section{Numerical calculations and discussions}

It is important to choose a numerical method of solving the Fredholm integral equation. The Fox and Goodwin methods require that the definite integrals should be calculable by numerical quadrature, using known formulae in the theory of finite differences, and Fredholm equations are conveniently treated by solving simultaneous equations. The methods enable accurate solutions to be obtained without a prohibitive expenditure of time and energy. The choice of an interval is of course rather arbitrary. We want to keep to a minimum number of linear equations, but the interval must not be large that the finite-difference equations are meaningless. Since the differences are examined, the method guards against the possibility of obtaining wrong results from this case. It also ensures that neither too few nor too many differences are retained in the quadrature formulae.

The method of Fox and Goodwin (1953) has been used to solve integral equation (3.12) numerically for different values of the dimensionless frequency $k_{s}$, material strip width $2 h$ and separating distance of the strips $c$. The integral in (3.12) has been represented by a quadrature formula involving values of the desired function $G$ at pivotal points in the range of integration, which leads to a set of algebraic linear simultaneous equations. The solution of the set of linear algebraic equations gives the first approximation of the required pivotal values of $G$ which has been improved by the use of the difference correction technique. After solving integral equation (3.12) for different values of engineering elastic constants of several orthotropic materials listed in Table 1 , the stress intensity factors (SIF), $k_{c}$ and $k_{1}$ at both ends of the strip given by equations (4.6) and (4.7) has been plotted against $k_{s}$ for different values of $h$ and $c$ and for different materials. Instead of the real part of SIF, its mod value is taken because both shows the same type of results.

In Fig. 2a and $4 \mathrm{a}, N_{c}$ (SIF, at the inner edge of the strip) and $N_{1}$ (SIF, at the outer edge of the strip) have been plotted against $k_{s}$ for $h=2.0$ and $h=2.5$ and for different strip lengths $(c=0.2,0.4,0.6)$ for material type I. In Fig. $3 \mathrm{a}$ and $5 \mathrm{a}, N_{c}$ and $N_{1}$ have been plotted against 
Table 1. Engineering elastic constants

\begin{tabular}{|l|l|c|c|c|c|}
\hline & & $E_{1}[\mathrm{~Pa}]$ & $E_{2}[\mathrm{~Pa}]$ & $\mu_{12}[\mathrm{~Pa}]$ & $\nu_{12}$ \\
\hline \hline Type I & E-type glass-epoxi composite & $9.79 \cdot 10^{9}$ & $42.3 \cdot 10^{9}$ & $3.66 \cdot 10^{9}$ & 0.063 \\
\hline Type II & Stainless steel-aluminium composite & $79.76 \cdot 10^{9}$ & $85.91 \cdot 10^{9}$ & $30.02 \cdot 10^{9}$ & 0.31 \\
\hline
\end{tabular}

$k_{s}$ for $c=0.4$ and $c=0.6$ and for different material strip widths $(h=2.0,2.5,3.0)$ for material type I. The same set of parameters stated above for the graphs of $N_{c}$ and $N_{1}$ have been plotted in Figs. 2b, 4b, 3b, 5b for material type II. For a particular value of material strip width $h$ $(=2.0,2.5)$, the value of $N_{c}$ decreases initially and, after increasing again, it decreases with an increase in $k_{s}$ for material type I (Fig. 2a), whereas for material type II, it is slowly decreasing with an increase in $k_{s}$ (Fig. $\left.2 \mathrm{~b}\right)$ for different values of strip length $c(=0.2,0.4,0.6)$. It is also observed that with an increase in $c$, the value of $N_{c}$ increases. When strip length $c$ is fixed, the value of $N_{c}$ is higher for higher values of $h(=2.0,2.5,3.0)$ (Fig. 3a and Fig. 3b) for both types of materials. Figure 4 and 5 show that $N_{1}$ has initial decreasing tendency and then increases with an increase in $k_{s}$ for both the materials. For fixed $c, N_{c}$ is higher when material strip width $h$ is higher. In all the cases, it is seen that as the length of the strip increases the value of $N_{1}$ decreases.

(a)

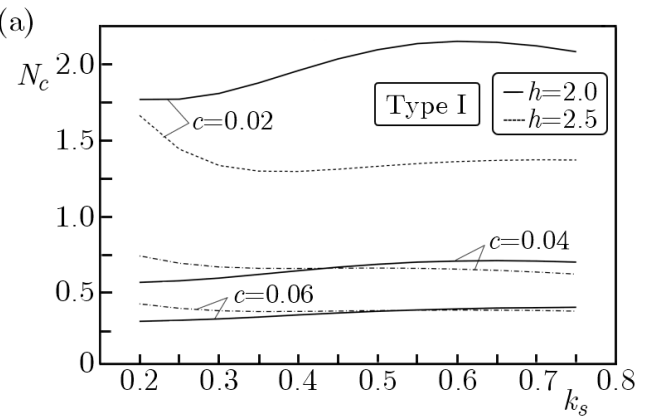

(b)

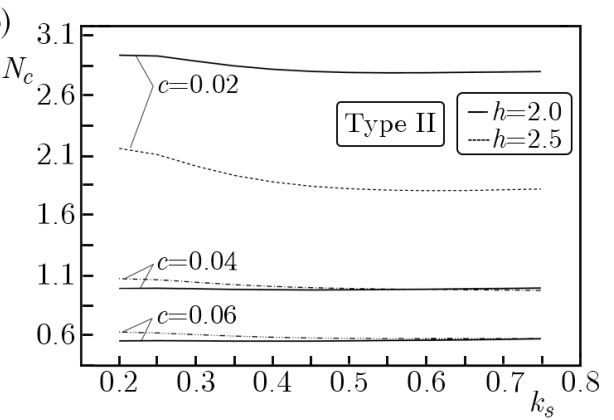

Fig. 2. Stress intensity factor $N_{c}$ verses frequency $k_{s}$

(a)

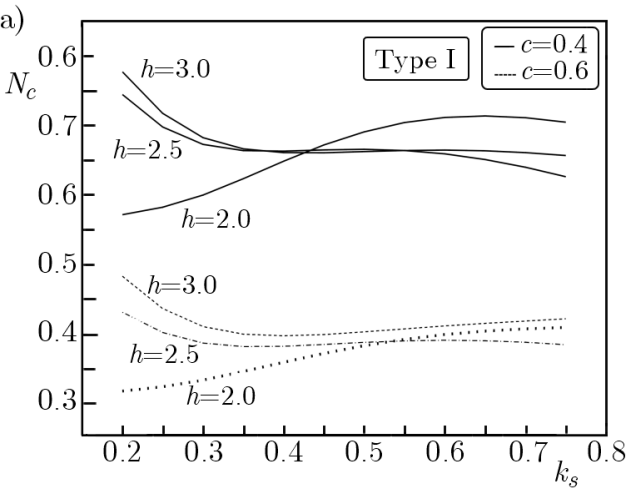

(b)

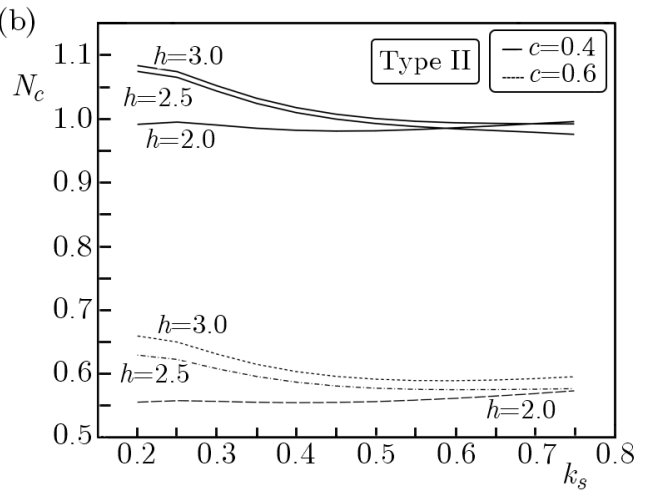

Fig. 3. Stress intensity factor $N_{c}$ verses frequency $k_{s}$

Finally, in Fig. 6 and 7 the vertical displacement $v(x, y)$ has been plotted outside the strips $(0<x<c, 1<x<h)$ for fixed values of $h=2.5, k_{s}=0.4$ and $c=0.6$ for both the materials. In Fig. 6, $v(x, y)$ has been plotted for the inner side of the strip $(0<x<c)$ and in Fig. 7 for the outer side of the strip $(1<x<h)$. In Fig. 6a and $7 \mathrm{a}$, it is observed that the vertical displacement $v(x, y)$ increases initially with an increment of the values of $x$ and $y$, then it decreases for material I. But in the case of Fig. $6 \mathrm{~b}$ and $7 \mathrm{~b}$, it is seen that the vertical displacement $v(x, y)$ increases slowly with an increase in the values of $x$ and $y$, then it decreases 
(a)

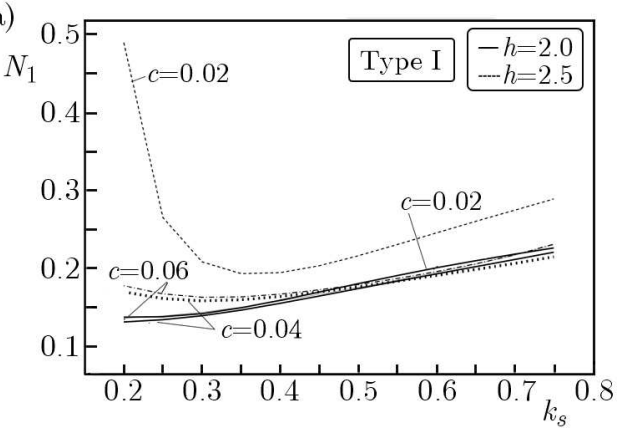

(b)

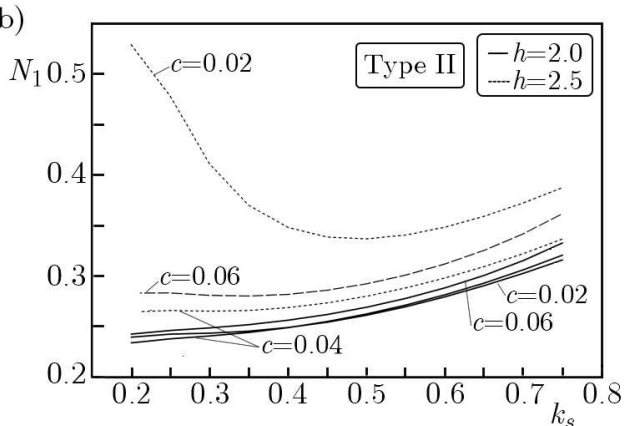

Fig. 4. Stress intensity factor $N_{1}$ versus frequency $k_{s}$

(a)

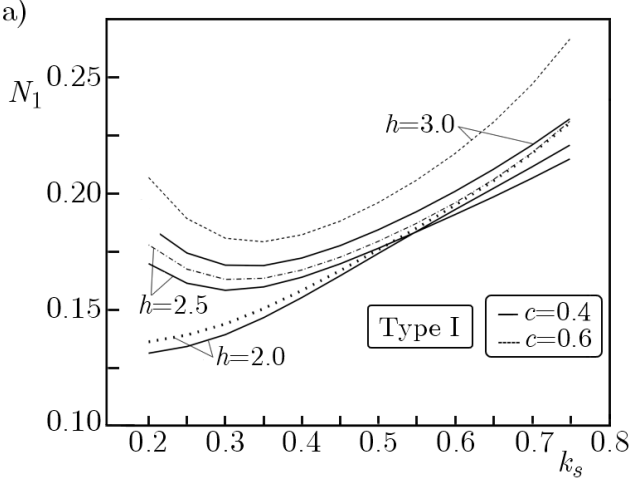

(b)

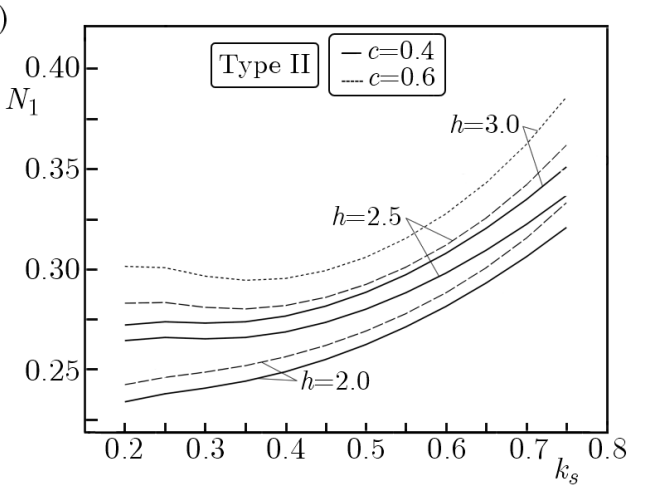

Fig. 5. Stress intensity factor $N_{1}$ versus frequency $k_{s}$

(a)

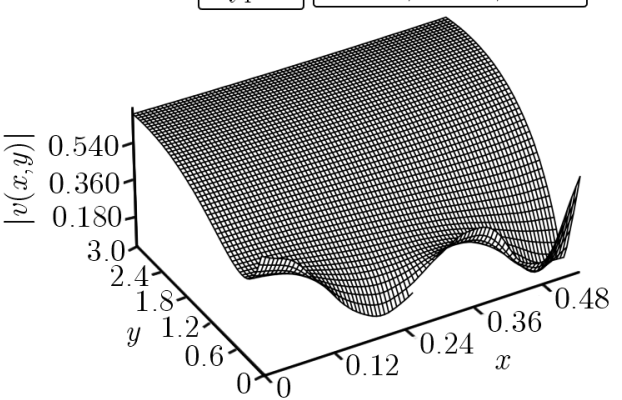

(b)

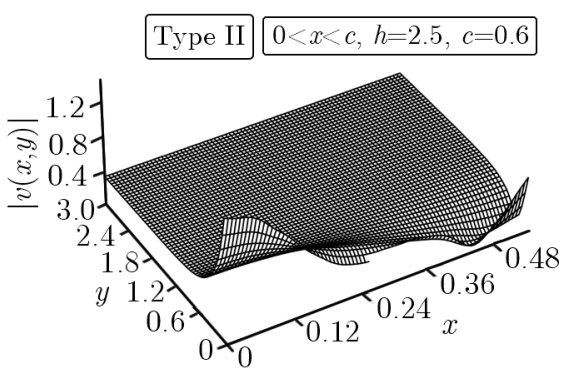

Fig. 6. Displacement $|v(x, y)|$ versus distances $(x, y)$

(a)

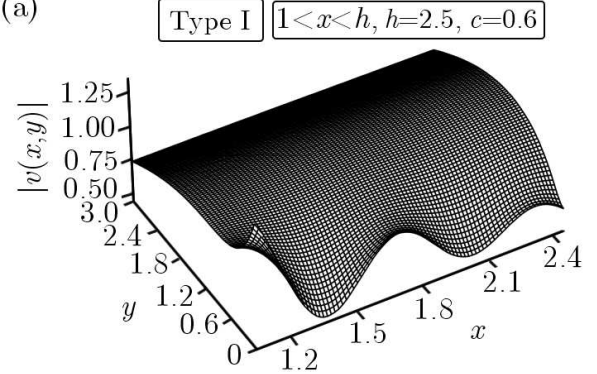

(b)

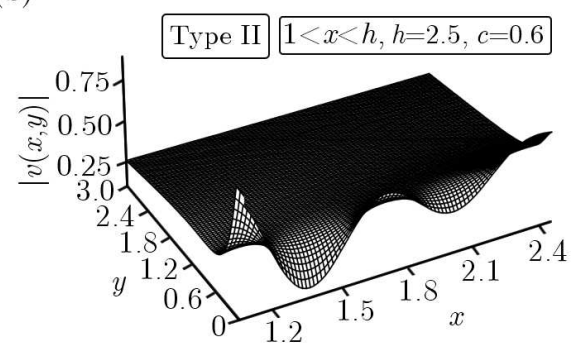

Fig. 7. Displacement $|v(x, y)|$ versus distances $(x, y)$ 
for material II. In all cases, the wave like nature has been observed, and finally the displacement tends to zero as $(x, y) \rightarrow \infty$, which satisfies the radiation condition.

\section{Conclusions}

The diffraction of the elastic $P$-wave by two rigid strips embedded in an infinite orthotropic strip is investigated on two types of materials by using the integral equation technique. The governing differential equation with constant coefficients with the boundary conditions becomes a mixed boundary value problem. Then, the mixed boundary value problem is transformed into a pair of dual integral equations with an unknown constant $A(\xi)$. To reduce the dual integral equations $(2.17)_{1}$ and $(2.17)_{2}$ to a single Fredholm integral equation, we assume the unknown constant $A(\xi)$ in the form of equation (3.1), so that equation $(2.17)_{2}$ can be automatically satisfied. Also, it has been found that the normal stress component $\tau_{y y}(x, 0)$ at the two tips of the strip has a square root singularity at $x=c$ and $x=1$. The form of (3.1) has a square root type singularity in it, which can be utilized to find stress singularities at the tips of the strips.

From all the graphs of SIF, it can be concluded that the SIF decreases gradually with an increment of the frequency $\left(k_{s}\right)$, after reaching the minimum value, it increases slowly. In all suggested cases, it is noted that the maximum value of the SIF at both tips of the strip for material II is little higher than that for material I. The SIF can be arrested within a certain range, which is very important with respect to growth of the crack. Finally, the vertical displacement $v(x, y)$ has been calculated outside the strips for both the materials. It has been observed the wave like nature from all the 3D figures, which finally decreases as the distance increases.

\section{Acknowledgement}

This research has been supported by the project "Mobile Computing and Innovative Applications" under UPE-II Programme of Jadavpur University. Also we thank the referees for their valuable comments to improve our paper.

\section{References}

1. Chen E.P., 1978, Sudden appearance of a crack in a stretched finite strip, Journal of Applied Mechanics, 45, 2, 277-280

2. Cinar A., Erdogan F., 1983, The crack and wedging problem for an orthotropic strip, International Journal of Fracture, 19, 83-102

3. DAS S., 2002, Interaction between line cracks in an orthotropic layer, International Journal Mathematics and Mathematical Sciences, 29, 2, 31-42

4. Das S., Chakraborty S., Srikanth N., Gupta M., 2008, Symmetric edge cracks in an orthotropic strip under normal loading, International Journal of Fracture, 153, 77-84

5. Das S., Patra B., Debnath L., 1998, Stress intensity factors for an interfacial crack between an orthotropic half-plane bonded to a dissimilar orthotropic layer with a punch, Computers and Mathematics with Applications, 35, 12, 27-40

6. Fox L., Goodwin E.T., 1953, The numerical solution of non-singular linear integral equations, Philosophical Transactions A, 245, 501-534

7. Iтоu S., 2012, Stress intensity factors for two parallel interface cracks between a nonhomogeneous bonding layer and two dissimilar orthotropic half-planes under tension, International Journal of Fracture, 175, 187-192

8. Li X.L., 2005, Two perfectly-bonded dissimilar orthotropic strips with an interfacial crack normal to the boundaries, Applied Mathematics and Computation, 163, 961-975 
9. Mandal S.C., Ghosh M.L., 1992, Forced vertical vibration of two rigid strips on a semi-infinite elastic solid, Journal of Sound and Vibration, 158, 1, 169-179

10. Mandal S.C., Ghosh M.L., 1994, Interaction of elastic waves with a periodic array of coplanar Griffith crack in an orthotropic medium, International Journal of Engineering Science, 32, 1, $167-178$

11. Mandal S.C., Pal S.C, Ghosh M.L., 1997, Forced vertical vibration of four rigid strips on semi-infinite elastic solid, International Journal of Solids and Structures, 34, 1017-1033

12. Mandal S.C., Pal S.C, Ghosh M.L., 1998, Diffraction of elastic waves by four coplanar rigid strips embedded in an infinite orthotropic medium, Journal of Engineering Mathematics, 33, 47-60

13. Monfared M.M., Ayatollahi M., 2013, Dynamic stress intensity factors of multiple cracks in an orthotropic strip with FGM coating, Engineering Fracture Mechanics, 109, 45-57

14. Morteza E.G., Ammar M., Azizollah A.B., 2010a, Rocking vibration of a rigid circular disc in a transversely isotropic full-space, International Journal for Numerical and Analytical Methods in Geomechanics (Wiley online Library)

15. Morteza E.G., Morteza F., Azizollah A.B., 2010b, Forced vertical vibration of rigid circular disc on a transversely isotropic half-space, Journal of Engineering Mechanics, 136, 913-922

16. MukherJee S., DAS S., 2007, Interaction of three interfacial Griffith cracks between bonded dissimilar orthotropic half planes, International Journal of Solids and Structures, 44, 5437-5446

17. Pramanick R.K., Pal S.C., Ghosh M.L., 1999, Shear wave interaction with a pair of rigid strips embedded in an infinitely long elastic strip, Journal of Technical Physics, 39, 31-44

18. Sarkar J., Ghosh M.L., Mandal S.C., 1995, Diffraction of elastic waves by two parallel rigid strips embedded in an infinite orthotropic medium, International Journal of Engineering Science, 33, 13, 1943-1958

19. Sarkar J., Mandal S.C., Ghosh M.L., 1995, Diffraction of elastic waves by three coplanar Griffith cracks in an orthotropic medium, International Journal of Engineering Science, 33, 2, 163-177

20. Siн G.C., 1977, Elastodynamic Crack Problems in Mechanics of Fracture, Noordhoff Leyden, 4

21. Sin G.C., Chen E.P., 1981, Cracks in Composite Materials, Martinus Nijhoff Publishers, 6

22. Singh B.M., Rokne J., Dhaliwal R.S., 1983, Diffraction of torsional wave by a circular rigid disc at the interface of two bonded dissimilar elastic solids, Acta Mechanica, 49, 139-146

23. Sinharoy S., 2013, Elastostatic problem of an infinite row of parallel cracks in an orthotropic medium under general loading, International Journal of Physics and Mathematical Sciences, 3, 1, 96-108

24. Wickнам G.R., 1977, The forced two dimensional oscillations of a rigid strip in smooth contact with a semi-infinite elastic solid, Mathematical Proceedings of the Cambridge Philosophical Society, 81, 291-311

25. Wu D., CAI Y., Xu C., Zhan H., 2006, Torsional vibrations of rigid circular plate on transversely isotropic saturated soil, Applied Mathematics and Mechanics, 27, 1541-1548 